\title{
Outbreaks of Foodborne Illness Associated with Melons ${ }^{1}$
}

\author{
Clara Diekman, Matthew D. Krug, Ashley T. Myers, Rachel McEgan, Keith R. Schneider, and \\ Michelle D. Danyluk²
}

This document is intended to serve as a reference for everyone interested in the safety of melons by providing a comprehensive list of melon-related outbreaks in the United States.

In recent years, foodborne illness outbreaks have become increasingly associated with produce (Walsh et al. 2014). One such commodity linked to foodborne illness are melons (specifically cantaloupe, honeydew, and watermelon), which are consumed around the world. Melons can be eaten alone or combined with other fruit and vegetables to make salads. Regardless of the way they are prepared, melons are typically consumed raw. This lack of a processing step increases the risk a foodborne pathogen making it to the consumer (Carstens et al. 2019).

While in the field, melons may be in direct contact with the soil, irrigation water, and insects, all of which are a potential source of contamination (Carstens et al. 2019; Walsh et al. 2014). Melons may also becom contaminated with foodborne pathogens during harvesting, packing, shipping, or preparation for consumption. During harvesting, mechanical damage resulting in wounds (e.g., punctures, cracks, bruising) may allow pathogen entry into the melon mesocarp tissue (i.e., edible flesh of the fruit) (US Food and Drug Administration 2013). Pathogen infiltration and adherence at the stem scar tissue (the end of the melon where the vine was removed), especially in cantaloupe, also can be problematic for food safety (US Food and Drug Administration 2013).

The characteristics and maturity of the melons can also influence the susceptibility to foodborne pathogen contamination. The characteristics of the melon rind, specifically the netted surfaces (cantaloupes), create a favorable environment for bacteria to grow and make it difficult to remove pathogens (Fu et al. 2020; Walsh et al. 2014). The maturity of the melon also can play a role in susceptibility because ripe melons may allow for better growth and survival of pathogens on their surfaces (Suslow 1997). Contamination on the surface of a melon may then be able to spread to the inside of the fruit once the melon is cut (Walsh et al. 2014).

This document serves as a reference for those concerned about the safety of melons, including cantaloupe, honeydew, and watermelon in the fresh and fresh-cut market. Outbreaks associated with melons in the United States, Canada, and Europe are highlighted with information regarding the location, pathogen, and incidence of illness.

1. This document is FSHN14-11, one of a series of the Food Science and Human Nutrition Department, UF/IFAS Extension. Original publication date November 2014. Revised August 2021. Visit the EDIS website at https://edis.ifas.ufl.edu for the currently supported version of this publication.

2. Clara Morgan Diekman, graduate student, Food Science and Human Nutrition Department, UF/IFAS Citrus Research and Education Center, Lake Alfred, FL; Matthew D Krug, state specialized agent, food science, UF/IFAS Southwest Florida Research and Education Center, Immokalee, FL; Ashley T. Myers, former graduate student, Food Science and Human Nutrition Department, UF/IFAS Citrus Research and Education Center, Lake Alfred, FL; Rachel McEgan, former research assistant, Food Science and Human Nutrition Department, UF/IFAS Citrus Research and Education Center, Lake Alfred, FL; Keith R. Schneider, professor, Food Science and Human Nutrition Department; and Michelle D. Danyluk, professor, Food Science and Human Nutrition Department, UF/IFAS Citrus Research and Education Center, Lake Alfred, FL; UF/IFAS Extension, Gainesville, FL 32611.

The Institute of Food and Agricultural Sciences (IFAS) is an Equal Opportunity Institution authorized to provide research, educational information and other services

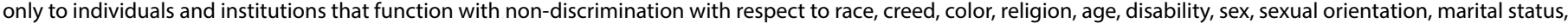

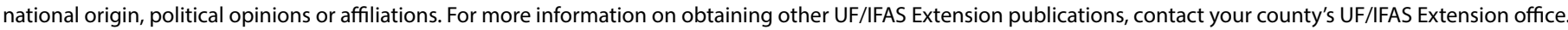
U.S. Department of Agriculture, UF/IFAS Extension Service, University of Florida, IFAS, Florida A \& M University Cooperative Extension Program, and Boards of County Commissioners Cooperating. Nick T. Place, dean for UF/IFAS Extension. 
Six tables are presented, representing different melon types (Table 1, cantaloupe; Table 2, honeydew; and Table 3, watermelon), unspecified melon and mixed fruits including melon (Table 4), outbreaks suspected to be associated with different melon types, unspecified melon, and mixed fruits including melon (Table 5), and outbreaks associated with multiple foods including melons (Table 6).

\section{References}

Bolstein, J. 1993. "An Outbreak of Salmonella Joviana Associated with Consumption of Watermelon." Environmental Health 56 (1): 29-31. https://www.jstor.org/stable/44534542

Carstens, C. K., J. K. Salazar, and C. Darkoh. 2019. "Multistate Outbreaks of Foodborne Illness in the United States Associated with Fresh Produce from 2010 to 2017." Frontiers in Microbiology 10 (November): 1-15. https://doi. org/10.3389/fmicb.2019.02667

Centers for Disease Control and Prevention (CDC). 1979. "Salmonella Oranienburg Gastroenteritis Associated with Consumption of Precut Watermelons: Illinois." Morb. Mortal. Wkly. Rep. 28:522-523.

Centers for Disease Control and Prevention (CDC). 1991. "Epidemiological Notes and Reports Multistate Outbreak of Salmonella Poona Infections-United States and Canada, 1991." Morb. Mortal. Wkly. Rep. 40:549-552. Last accessed on 3-9-21. https://www.cdc.gov/mmwr/preview/ mmwrhtml/00014966.htm

Centers for Disease Control and Prevention (CDC). 2002. "Multistate Outbreaks of Salmonella Serotype Poona Infections Associated with Eating Cantaloupe from MexicoUnited States and Canada, 2000-2002." Morb. Mortal. Wkly. Rep. 51:1044-1047. Last accessed on 3-9-21. https://www. cdc.gov/mmwr/preview/mmwrhtml/mm5146a2.htm

Centers for Disease Control and Prevention (CDC). 2007. "Salmonella Oranienburg Infections Associated with Fruit Salad Served in Health-Care Facilities-Northeastern United States and Canada, 2006." Morb. Mortal. Wkly. Rep. 56:1025-1028. Last accessed on 3-9-21. https://www.cdc. gov/mmwr/preview/mmwrhtml/mm5639a3.htm

Centers for Disease Control and Prevention (CDC). 2008a. "Multistate Outbreak of Salmonella Litchfield Infections Linked to Cantaloupe (Final Update)." Last accessed on 3-16-20. https://www.cdc.gov/salmonella/2008/cantaloupes-4-2-2008.html
Centers for Disease Control and Prevention (CDC). 2008b. "Salmonella Litchfield Outbreak Associated with a Hotel Restaurant-Atlantic City, New Jersey, 2007." Morb. Mortal. Wkly. Rep. 57:775-779. Last accessed on 3-9-21. https:// www.cdc.gov/mmwr/preview/mmwrhtml/mm5728a4.htm

Centers for Disease Control and Prevention (CDC). 2018. National Outbreak Reporting System (NORS). Last accessed on 3-9-21. https://wwwn.cdc.gov/norsdashboard/

Deeks, S., A. Ellis, B. Ciebin, R. Khakhria, M. Naus, and J. Hockin. 1998. “Salmonella Oranienburg, Ontario." Can. Comm. Dis. Rep. 24:177-179.

Del Rosario, B. A., and L. R. Beuchat. 1995. "Survival and Growth of Enterohemorrhagic Escherichia coli O157:H7 in Cantaloupe and Watermelon." Journal of Food Protection 58 (1): 105-107. https://doi.org/10.4315/0362-028X-58.1.105

Food and Agriculture Organization of the United Nations (FAO). 2011. "Microbial Hazards and Melons: Codex Committee on Food Hygiene Working Group on the Development of an Annex on Methods for the Code of Hygienic Practice for Fresh Fruits and Vegetables (CAC/ RCP 53-2003)." Last accessed on 3-9-21. https://www.who. int/foodsafety/publications/micro/MRA_FruitVeges.pdf

Fu, Y., A. K. Bhunia, and Y. Yao. 2020. "Abrasive Brushing Reduces Pathogen Biofilms at Cantaloupe Rind Surface." In International Journal of Food Microbiology 329:108685. https://doi.org/10.1016/j.ijfoodmicro.2020.108685

Gayler, G. E., R. A. MacCready, J. P. Reardon, and B. F. McKernan. 1995. "An Outbreak of Salmonellosis Traced to Watermelon." Public Health Reports 70 (3): 311-313. Last accessed on 3-9-21. https://www.ncbi.nlm.nih.gov/pmc/ articles/PMC2024507/

McCollum, J. T., A. B. Cronquist, B. J. Silk, K. A. Jackson, K. A. O'Connor, S. Cosgrove, J. P. Gossack, et al. 2013. "Multistate Outbreak of Listeriosis Associated with Cantaloupe." New England Journal of Medicine 369 (10): 944-953. https:// doi.org/10.1056/nejmoa1215837

Mohle-Boetani, J. C., R. Reporter, S. Benson Werner, S. Abbott, J. Farrar, S. H. Waterman, and D. J. Vugia. 1999. "An Outbreak of Salmonella Serogroup Saphra Due to Cantaloupes from Mexico." Journal of Infectious Diseases 180 (4): 1361-1364. https://doi.org/10.1086/314995 
Nielsen, C. F., A. Langer, J. Pringle, R. Heffernan, R. Klos, T. Monson, M. Rauch, J. Ball, M. Hoekstra, J. Archer, M. Sotir, and J. Davis. 2010. "First Documented Multistate Outbreak of Salmonella Carrau Infections-United States, 2009."

Abstract. 59th Annual Epidemic Intelligence Service. 19-23 April 2010. Last accessed on 3-9-21. http://www.cdc.gov/ eis/downloads/2010.EIS.Conference.pdf

Public Health Agency of Canada (PHAC). 2009.

"Update: Salmonella Carrau Outbreak." Last accessed on 3-9-21. http://www.phac-aspc.gc.ca/new-nouv-eng. php? year $=2009 \&$ month $=$ may

Ries, A. A., S. Zaza, and C. Langkop. 1990. "A Multistate Outbreak of Salmonella Chester Linked to Imported Cantaloupe (Abstract)." In American Society for Microbiology Program and Abstracts of the $30^{\text {th }}$ Interscience Conference on Antimicrobial Agents and Chemotherapy, 238. Washington, DC: American Society for Microbiology.

Suslow, T. V. 1997. "Postharvest Chlorination Basic Properties and Key Points for Effective Disinfection1997." Publication 8003. University of California, Davis Division of Agriculture and Natural Resources. Last accessed on 3-9-21. http://ucanr.edu/datastoreFiles/234-404.pdf

US Food and Drug Administration (FDA). 2009. "Chapter IV: Outbreaks Tables, Analysis and Evaluation of Preventive Control Measures of the Control and Reduction/Elimination of Microbial Hazards on Fresh and Fresh-Cut Produce; Outbreaks Associated with Fresh Produce: Incidence, Growth, and Survival of Pathogens in Fresh and Fresh-Cut Produce." Last accessed 3-9-21. http://wayback.archive-it. org/7993/20170111183953/http:/www.fda.gov/Food/ FoodScienceResearch/SafePracticesforFoodProcesses/ ucm091265.htm

US Food and Drug Administration. 2013. "National Commodity-Specific Food Safety Guidelines for Cantaloupes and Netted Melons." Last accessed 3-9-21. https://www.fda. gov/food/guidance-documents-regulatory-informationtopic-food-and-dietary-supplements/produce-plantproducts-guidance-documents-regulatory-information

Walsh, K. A., S. D. Bennett, M. Mahovic, and L. H. Gould. 2014. "Outbreaks Associated with Cantaloupe, Watermelon, and Honeydew in the United States, 1973-2011." Foodborne Pathogens and Disease 11 (12): 945-952. Last accessed on 3-16-21. https://doi.org/10.1089/fpd.2014.1812 
Table 1. Outbreaks of foodborne disease associated with cantaloupe, 1997-2016*

\begin{tabular}{|c|c|c|c|c|c|c|c|}
\hline Year & Month & Location & Pathogen $^{a}$ & $\begin{array}{l}\text { Location of } \\
\text { Consumption }^{b}\end{array}$ & $\begin{array}{c}\text { Cases } \\
\text { (Deaths) }^{b}\end{array}$ & $\begin{array}{l}\text { Food } \\
\text { Vehicle }\end{array}$ & References \\
\hline 1997 & Feb-May & US (CA) & $\begin{array}{l}\text { Salmonella } \\
\text { Saphra }\end{array}$ & $\begin{array}{l}\text { Private home, } \\
\text { grocery store, } \\
\text { restaurant }\end{array}$ & $24(0)$ & Cantaloupe & Mohle-Boetani et al. 1999 \\
\hline 2000 & April-June & US (multistate) & Salmonella Poona & $\begin{array}{l}\text { Restaurant-other } \\
\text { or unknown }\end{array}$ & $47(0)$ & Cantaloupe & CDC 2018 \\
\hline 2001 & April & US (OR) & Salmonella & $\begin{array}{l}\text { Restaurant-other } \\
\text { or unknown }\end{array}$ & $2(0)$ & Cantaloupe & CDC 2018 \\
\hline 2001 & April-May & US (multistate) & Salmonella Poona & $\begin{array}{l}\text { Grocery store; } \\
\text { other }\end{array}$ & $50(2)$ & Cantaloupe & CDC 2018 \\
\hline 2002 & April & US (multistate) & Salmonella Poona & $\begin{array}{l}\text { Private home/ } \\
\text { residence; grocery } \\
\text { store; long-term } \\
\text { care/nursing } \\
\text { home/assisted- } \\
\text { living facility }\end{array}$ & 48 (NR) & Cantaloupe & CDC 2018 \\
\hline 2007 & December & US (multistate) & $\begin{array}{l}\text { Salmonella } \\
\text { Litchfield }\end{array}$ & $\begin{array}{l}\text { Restaurant-other } \\
\text { or unknown }\end{array}$ & $53(0)$ & Cantaloupe & CDC 2018 \\
\hline 2008 & November & US (multistate) & $\begin{array}{l}\text { Salmonella } \\
\text { Javiana }\end{array}$ & NR & $10(\mathrm{NR})$ & Cantaloupe & CDC 2018 \\
\hline 2008 & December & US (CA) & Norovirus & Restaurant & $23(0)$ & Cantaloupe & CDC 2018 \\
\hline 2011 & February & US (multistate) & $\begin{array}{l}\text { Salmonella } \\
\text { Panama }\end{array}$ & Private home & $20(0)$ & Cantaloupe & CDC 2018 \\
\hline 2011 & June & US (multistate) & $\begin{array}{l}\text { Salmonella } \\
\text { Uganda }\end{array}$ & NR & $25(0)$ & Cantaloupe & CDC 2018 \\
\hline 2011 & July & US (multistate) & L. monocytogenes & Private home & $147(33)$ & Cantaloupe & CDC 2018 \\
\hline 2011 & August-October & US (multistate) & L. monocytogenes & Grocery retailer & $147(33)$ & Cantaloupe & McCollum et al. 2013 \\
\hline 2012 & July & US (multistate) & $\begin{array}{l}\text { Salmonella } \\
\text { Newport }\end{array}$ & $\begin{array}{l}\text { Private home/ } \\
\text { residence }\end{array}$ & $24(0)$ & Cantaloupe & CDC 2018 \\
\hline 2012 & July & US (multistate) & $\begin{array}{l}\text { Salmonella } \\
\text { Newport }\end{array}$ & $\begin{array}{l}\text { Private home/ } \\
\text { residence }\end{array}$ & $33(1)$ & Cantaloupe & CDC 2018 \\
\hline 2012 & July & US (multistate) & $\begin{array}{l}\text { Salmonella } \\
\text { Typhimurium }\end{array}$ & NR & $14(0)$ & Cantaloupe & CDC 2018 \\
\hline 2012 & July-September & US (multistate) & $\begin{array}{l}\text { Salmonella } \\
\text { Typhimurium } \\
\text { and Salmonella } \\
\text { Newport }\end{array}$ & $\begin{array}{l}\text { Private home/ } \\
\text { residence; all } \\
\text { sourced from same } \\
\text { farm }\end{array}$ & $261(3)$ & Cantaloupe & CDC 2018 \\
\hline 2013 & April & US (IN) & $\begin{array}{l}\text { Salmonella } \\
\text { Typhimurium } \\
\text { and Salmonella } \\
\text { Typhimurium }\end{array}$ & NR & $14(0)$ & Cantaloupe & CDC 2018 \\
\hline 2014 & April & US (multistate) & $\begin{array}{l}\text { Salmonella } \\
\text { Baildon }\end{array}$ & $\begin{array}{l}\text { Private home/ } \\
\text { residence }\end{array}$ & $20(0)$ & Cantaloupe & CDC 2018 \\
\hline 2016 & June & US (multistate) & $\begin{array}{l}\text { Salmonella } \\
\text { Minnesota }\end{array}$ & $\begin{array}{l}\text { Restaurant-Sit- } \\
\text { down dining; } \\
\text { private home/ } \\
\text { residence; grocery } \\
\text { store }\end{array}$ & $10(0)$ & Cantaloupe & CDC 2018 \\
\hline
\end{tabular}

${ }^{\text {a }}$ Pathogens abbreviated and associated with outbreaks include E. (Escherichia), L. (Listeria)

${ }^{\mathrm{b}}$ NR: Not Reported 
Table 2. Outbreaks of foodborne disease associated with honeydew, 2003*

\begin{tabular}{|c|c|c|c|c|c|c|c|}
\hline Year & Month & Location & Pathogen & Location of Consumption & $\begin{array}{c}\text { Cases } \\
\text { (Deaths) }\end{array}$ & $\begin{array}{c}\text { Food } \\
\text { Vehicle }\end{array}$ & References \\
\hline 2003 & January & US (multistate) & $\begin{array}{l}\text { Salmonella } \\
\text { Newport }\end{array}$ & $\begin{array}{l}\text { Restaurant-other or unknown type; } \\
\text { private home/residence; caterer (food } \\
\text { prepared off-site from where served); } \\
\text { grocery store; long-term care/nursing } \\
\text { home/assisted living facility; hospital }\end{array}$ & $68(2)$ & Honeydew & CDC 2018 \\
\hline 2003 & September & US (CO) & Shigella sonnei & Restaurant-other or unknown & $56(0)$ & Honeydew & CDC 2018 \\
\hline
\end{tabular}

*Table was last updated on 5-3-2021. The most recent data published by the CDC indicates that there have been no outbreaks since 2003.

Table 3. Outbreaks of foodborne disease associated with watermelon, 1950-2017*

\begin{tabular}{|c|c|c|c|c|c|c|c|}
\hline Year & Month & Location & Pathogen ${ }^{a}$ & Location of Consumption & $\begin{array}{c}\text { Cases } \\
\text { (Deaths) }\end{array}$ & Food Vehicle & References \\
\hline 1950 & $N R^{c}$ & US (MN) & Salmonella Bareilly & Roadside stand & $6(0)$ & Watermelon, cut & Gayler et al. 1955 \\
\hline 1954 & June & US (MA) & Salmonella Miami & Supermarket & $17(1)$ & Watermelon, cut & Gayler et al. 1955 \\
\hline 1979 & NR & US (IL) & $\begin{array}{l}\text { Salmonella } \\
\text { Oranienburg }\end{array}$ & Supermarket & $6(0)$ & Watermelon & CDC 1979 \\
\hline 1987 & NR & Sweden & Shigella sonnei & Dinner party & $15(0)$ & Watermelon & FAO 2011 \\
\hline 1991 & June & US (MI) & Salmonella Javiana & $\begin{array}{l}\text { Indoor picnic/school party; grocery } \\
\text { retailer }\end{array}$ & $39(0)$ & Watermelon & Bolstein 1993 \\
\hline 1993 & NR & US & Salmonella Javiana & Private home, church & $27(0)$ & Watermelon & $\begin{array}{l}\text { Del Rosario and } \\
\text { Beuchat } 1995\end{array}$ \\
\hline 2000 & July & US (WI) & E. coli O157:H7 & Restaurant & $736(1)$ & Watermelon & CDC 2018 \\
\hline 2005 & July & US (ID) & Norovirus & Camp & $18(0)$ & Watermelon & CDC 2018 \\
\hline 2006 & July & US (CA) & Norovirus & Grocery store & $14(0)$ & Watermelon & CDC 2018 \\
\hline 2006 & July & US (VA) & Camplyobacter jejuni & Other & $15(0)$ & Watermelon & CDC 2018 \\
\hline 2006 & August & US (NY) & Salmonella Newport & Restaurant & $20(0)$ & Watermelon & CDC 2018 \\
\hline 2008 & October & US (CA) & Salmonella Javiana & Other & $594(0)$ & Watermelon & CDC 2018 \\
\hline 2010 & July & US (MI) & $\begin{array}{l}\text { Salmonella } \\
\text { Saintpaul }\end{array}$ & $\begin{array}{l}\text { Private home/residence; grocery } \\
\text { store }\end{array}$ & $17(0)$ & Watermelon & CDC2018 \\
\hline 2011 & August & US (MN) & $\begin{array}{l}\text { Salmonella } \\
\text { Typhimurium }\end{array}$ & Private home/residence & $15(0)$ & Watermelon & CDC 2018 \\
\hline 2017 & June & $\begin{array}{l}\text { US } \\
\text { (Multistate) }\end{array}$ & Salmonella Newport & Unknown & $20(0)$ & Watermelon & CDC 2018 \\
\hline 2017 & July & US (PA) & Salmonella Newport & Private home/residence & $13(0)$ & Watermelon & CDC 2018 \\
\hline
\end{tabular}

a Pathogens abbreviated and associated with outbreaks include E. (Escherichia)

*Table was last updated on 5-3-2021. The most recent data published by the CDC indicates that there have been no outbreaks since 2017. 
Table 4. Foodborne Illness Outbreaks Associated with Unspecified Melons and Mixed Fruit (including melon), 1998-2019*

\begin{tabular}{|c|c|c|c|c|c|c|c|}
\hline Year & Month & Location & Pathogen & Location of Consumption & $\begin{array}{c}\text { Cases } \\
\text { (Deaths) }\end{array}$ & Food Vehicle & References \\
\hline 1998 & August & US (IA) & Norovirus & $\begin{array}{l}\text { Restaurant-other or unknown } \\
\text { type }\end{array}$ & $41(0)$ & Strawberries; honeydew & CDC 2018 \\
\hline 1999 & May & US (WI) & Norovirus & Restaurant & $23(0)$ & $\begin{array}{l}\text { Melon (unspecified), } \\
\text { pineapple, watermelon }\end{array}$ & CDC 2018 \\
\hline 1999 & July & US (CA) & $\begin{array}{l}\text { Salmonella } \\
\text { Enteritidis }\end{array}$ & School & $82(0)$ & Honeydew, watermelon & CDC 2018 \\
\hline 1999 & June & US (IA) & Norovirus & Restaurant & $61(0)$ & $\begin{array}{l}\text { Cantaloupe, honeydew, } \\
\text { watermelon }\end{array}$ & CDC 2018 \\
\hline 2000 & June & US (CO) & $\begin{array}{l}\text { Salmonella } \\
\text { Heidelberg }\end{array}$ & Restaurant & $4(0)$ & Melon (unspecified) & CDC 2018 \\
\hline 2001 & January & US (KS) & Norovirus & Restaurant & $36(0)$ & $\begin{array}{l}\text { Cantaloupe, honeydew, } \\
\text { pineapple }\end{array}$ & CDC 2018 \\
\hline 2001 & March & US (MN) & Norovirus & Office/indoor workplace & $42(0)$ & $\begin{array}{l}\text { Cantaloupe, pineapple, } \\
\text { unspecified }\end{array}$ & CDC 2018 \\
\hline 2001 & June & US (CA) & $\begin{array}{l}\text { Salmonella } \\
\text { Poona }\end{array}$ & $\begin{array}{l}\text { Daycare, picnic, private home, } \\
\text { restaurant }\end{array}$ & $23(0)$ & $\begin{array}{l}\text { Honeydew, musk melon, } \\
\text { watermelon }\end{array}$ & CDC 2018 \\
\hline 2002 & January & US (MN) & Norovirus & Restaurant & $15(0)$ & $\begin{array}{l}\text { Strawberries; melon, } \\
\text { unspecified; pineapple, } \\
\text { unspecified }\end{array}$ & CDC 2018 \\
\hline 2002 & September & US (WA) & $\begin{array}{l}\text { Salmonella } \\
\text { Berta }\end{array}$ & $\begin{array}{l}\text { Caterer (food prepared off-site } \\
\text { from where served) }\end{array}$ & $29(0)$ & $\begin{array}{l}\text { Cantaloupe, grapes, } \\
\text { watermelon }\end{array}$ & CDC 2018 \\
\hline 2003 & May & $\begin{array}{l}\text { US } \\
\text { (multistate) }\end{array}$ & $\begin{array}{l}\text { Salmonella } \\
\text { Muenchen }\end{array}$ & $\begin{array}{l}\text { Daycare, private home, grocery } \\
\text { store }\end{array}$ & $58(0)$ & Cantaloupe, honeydew & CDC 2018 \\
\hline 2003 & August & US (FL) & Norovirus & Nursing home & $16(0)$ & $\begin{array}{l}\text { Cantaloupe, banana, } \\
\text { pineapple }\end{array}$ & CDC 2018 \\
\hline 2004 & June & US (WI) & Norovirus & Church & $34(0)$ & $\begin{array}{l}\text { Honeydew, musk melon, } \\
\text { watermelon }\end{array}$ & CDC 2018 \\
\hline 2004 & March & US (KS) & Norovirus & School/college/university & $100(0)$ & $\begin{array}{l}\text { Cantaloupe, honeydew, } \\
\text { watermelon }\end{array}$ & CDC 2018 \\
\hline 2008 & August & US (CO) & $\begin{array}{l}\text { Salmonella } \\
\text { Newport }\end{array}$ & Private home & $3(0)$ & Cantaloupe, watermelon & CDC 2018 \\
\hline 2011 & April & US (GA) & Norovirus & $\begin{array}{l}\text { Banquet Facility (food prepared } \\
\text { and served on-site) }\end{array}$ & $22(0)$ & $\begin{array}{l}\text { pineapple, unspecified; } \\
\text { cantaloupe }\end{array}$ & CDC 2018 \\
\hline 2011 & June & US (OR) & $\begin{array}{l}\text { Salmonella } \\
\text { Typhimurium }\end{array}$ & $\begin{array}{l}\text { Private home/residence; Child } \\
\text { day care }\end{array}$ & $6(0)$ & $\begin{array}{l}\text { Cantaloupe \& strawberry } \\
\text { mix }\end{array}$ & CDC 2018 \\
\hline 2015 & February & US (OH) & $\begin{array}{l}\text { Norovirus GII.4 } \\
\text { Sydney }\end{array}$ & $\begin{array}{l}\text { Restaurant-sit-down dining; } \\
\text { private home/residence }\end{array}$ & $35(0)$ & $\begin{array}{l}\text { Cantaloupe, pineapple, } \\
\text { unspecified; grapes }\end{array}$ & CDC 2018 \\
\hline 2016 & September & US (OR) & $\begin{array}{l}\text { Norovirus Gll.4 } \\
\text { untypeable }\end{array}$ & $\begin{array}{l}\text { Banquet facility (food prepared } \\
\text { and served on-site) }\end{array}$ & $26(0)$ & $\begin{array}{l}\text { Strawberries; cantaloupe; } \\
\text { honeydew }\end{array}$ & CDC 2018 \\
\hline 2017 & October & $\begin{array}{l}\text { US } \\
\text { (multistate) }\end{array}$ & $\begin{array}{l}\text { Salmonella } \\
\text { Newport }\end{array}$ & Unknown & $24(1)$ & Melon, unspecified & CDC 2018 \\
\hline 2018 & April & $\begin{array}{l}\text { US } \\
\text { (multistate) }\end{array}$ & $\begin{array}{l}\text { Salmonella } \\
\text { Adelaide }\end{array}$ & Other & $77(1)$ & Melon, unspecified & CDC 2018 \\
\hline 2019 & March-May & $\begin{array}{l}\text { US } \\
\text { (multistate) }\end{array}$ & $\begin{array}{l}\text { Salmonella } \\
\text { Carrau }\end{array}$ & Grocery store & $137(0)$ & $\begin{array}{l}\text { Watermelon, honeydew, } \\
\text { cantaloupe, fruit medley } \\
\text { (containing one of these } \\
\text { melons) }\end{array}$ & CDC 2019 \\
\hline 2019 & $\begin{array}{l}\text { November- } \\
\text { January }\end{array}$ & $\begin{array}{l}\text { US } \\
\text { (multistate) }\end{array}$ & $\begin{array}{l}\text { Salmonella } \\
\text { Javiana }\end{array}$ & $\begin{array}{l}\text { Long-term care facilities, } \\
\text { hospitals, hotels, schools, and } \\
\text { grocery stores }\end{array}$ & $165(0)$ & $\begin{array}{l}\text { Honeydew, cantaloupe, } \\
\text { pineapple, and grapes }\end{array}$ & CDC 2020 \\
\hline
\end{tabular}

*Table was last updated on 5-3-2021. The most recent data published by the CDC indicates that there have been no outbreaks since 2019. 
Table 5. Foodborne illness outbreaks suspected to be associated with cantaloupe, honeydew, or watermelon or with unspecified melons, or mixed fruit (including melon), 1987-2017*

\begin{tabular}{|c|c|c|c|c|c|c|c|}
\hline Year & Month & Location & Pathogena & $\begin{array}{l}\text { Location of } \\
\text { Consumption }^{b}\end{array}$ & $\begin{array}{c}\text { Cases } \\
\text { (deaths) }\end{array}$ & Suspected Food Vehicle & References \\
\hline 1987 & $N R^{b}$ & UK & Norovirus & NR & $206(0)$ & Melon (unspecified) & FDA 2009 \\
\hline 1990 & January & US (multistate) & $\begin{array}{l}\text { Salmonella } \\
\text { Chester }\end{array}$ & Restaurant salad bars & $245(2)$ & Cantaloupe & $\begin{array}{l}\text { Ries, Zaza, and } \\
\text { Langkop } 1990\end{array}$ \\
\hline 1991 & June & $\begin{array}{l}\text { US (IL, MI) and } \\
\text { Canada }\end{array}$ & $\begin{array}{l}\text { Salmonella } \\
\text { Poona }\end{array}$ & $\begin{array}{l}\text { Grocery stores, } \\
\text { restaurants }\end{array}$ & $400(0)$ & Cantaloupe & CDC 1991 \\
\hline 1993 & NR & US & $\begin{array}{l}\text { Campylobacter } \\
\text { jejuni }\end{array}$ & Food service & $48(0)$ & $\begin{array}{l}\text { Melon (unspecified), } \\
\text { strawberries }\end{array}$ & FAO 2011 \\
\hline 1997 & NR & US (OR) & E. coli O157:H7 & Restaurant & $9(0)$ & Cantaloupe & FDA 2009 \\
\hline 1998 & May-June & Canada (ON) & $\begin{array}{l}\text { Salmonella } \\
\text { Oranienburg }\end{array}$ & Supermarket & $22(0)$ & Cantaloupe & $\begin{array}{l}\text { Deeks et al. } \\
1998\end{array}$ \\
\hline 1998 & August & US (IA) & Norovirus & Restaurant & $14(0)$ & Honeydew, strawberries & CDC n.d. \\
\hline 1999 & September & US (MN) & Norovirus & Restaurant & $5(0)$ & Cantaloupe & CDC 2018 \\
\hline 2000 & May & US (IL) & $\begin{array}{l}\text { Bacilluscereus, } \\
\text { Staphylococcus } \\
\text { aureus }\end{array}$ & Church, temple & $55(0)$ & Melon (unspecified) & CDC 2018 \\
\hline 2001 & January & US (CO) & Norovirus & Restaurant & $100(1)$ & Honeydew, pineapple & CDC 2018 \\
\hline 2001 & March & US (MN) & Norovirus & Workplace & $42(0)$ & Cantaloupe, pineapple & CDC n.d. \\
\hline 2001 & March & US (FL) & NR & NR & $33(0)$ & Melon (unspecified) & CDC n.d. \\
\hline 2001 & June & US (WA) & NR & Restaurant & $4(0)$ & Cantaloupe, pineapple & CDC n.d. \\
\hline 2001 & December & US (OR) & Salmonella spp. & $\begin{array}{l}\text { Nursing home, home } \\
\text { care, restaurant }\end{array}$ & $2(0)$ & Cantaloupe & CDC n.d. \\
\hline 2002 & March-May & $\begin{array}{l}\text { US (multistate), } \\
\text { Canada }\end{array}$ & $\begin{array}{l}\text { Salmonella } \\
\text { Poona }\end{array}$ & $\begin{array}{l}\text { Nursing home, home } \\
\text { care, private home }\end{array}$ & $58(0)$ & Cantaloupe & CDC 2002 \\
\hline 2002 & June & US (DC) & $N R$ & NR & $11(0)$ & Watermelon, strawberries & CDC n.d. \\
\hline 2004 & NR & US (NR) & E. coli 0157:H7 & NR & $6(0)$ & Cantaloupe & FAO 2011 \\
\hline 2004 & April & US (CO) & Norovirus & $\begin{array}{l}\text { Nursing home, home } \\
\text { care }\end{array}$ & $62(0)$ & $\begin{array}{l}\text { Melon (unspecified), } \\
\text { house salad, strawberries }\end{array}$ & CDC n.d. \\
\hline 2004 & November & US (CA) & Norovirus & Other & $30(0)$ & $\begin{array}{l}\text { Cantaloupe, honeydew, } \\
\text { watermelon }\end{array}$ & CDC 2018 \\
\hline 2006 & April & US (CA) & Norovirus & Other & $108(0)$ & $\begin{array}{l}\text { Mixed fruit; sandwich, } \\
\text { beef }\end{array}$ & CDC 2018 \\
\hline 2006 & June-July & $\begin{array}{l}\text { US (multistate), } \\
\text { Canada (ON) }\end{array}$ & $\begin{array}{l}\text { Salmonella } \\
\text { Oranienburg }\end{array}$ & $\begin{array}{l}\text { Grocery store, health } \\
\text { care facility, nursing } \\
\text { home }\end{array}$ & $41(0)$ & $\begin{array}{l}\text { Cantaloupe, honeydew, } \\
\text { fruit salad }\end{array}$ & CDC 2007 \\
\hline 2007 & NR & US & $\begin{array}{l}\text { Salmonella } \\
\text { Litchfield }\end{array}$ & $\begin{array}{l}\text { Private home, } \\
\text { restaurant }\end{array}$ & $11(0)$ & Honeydew & FAO 2011 \\
\hline 2007 & February & US (MI) & NR & Banquet facility & $8(0)$ & Cantaloupe, watermelon & CDC n.d. \\
\hline 2007 & May-June & US (NJ) & $\begin{array}{l}\text { Salmonella } \\
\text { Litchfield }\end{array}$ & Hotel restaurant & $30(0)$ & Fruit salad, honeydew & CDC 2008b \\
\hline 2007 & September & US (MN) & Norovirus & Private home & $12(0)$ & $\begin{array}{l}\text { Grapes, unspecified; } \\
\text { pineapple, unspecified; } \\
\text { watermelon; cake, } \\
\text { unspecified }\end{array}$ & CDC 2018 \\
\hline 2008 & January-March & $\begin{array}{l}\text { US (multistate), } \\
\text { Canada }\end{array}$ & $\begin{array}{l}\text { Salmonella } \\
\text { Litchfield }\end{array}$ & $\begin{array}{l}\text { Hospital, private } \\
\text { home }\end{array}$ & $51(0)$ & Cantaloupe & CDC 2008a \\
\hline 2008 & June & US (MN) & Norovirus & $\begin{array}{l}\text { Private home, } \\
\text { grocery store }\end{array}$ & $3(0)$ & $\begin{array}{l}\text { Brie, unspecified; sauces, } \\
\text { unspecified; pineapple, } \\
\text { unspecified; cantaloupe }\end{array}$ & CDC 2018 \\
\hline
\end{tabular}




\begin{tabular}{|c|c|c|c|c|c|c|c|}
\hline Year & Month & Location & Pathogena & $\begin{array}{l}\text { Location of } \\
\text { Consumption }^{b}\end{array}$ & $\begin{array}{c}\text { Cases } \\
\text { (deaths) }\end{array}$ & Suspected Food Vehicle & References \\
\hline 2009 & February & US (multistate) & $\begin{array}{l}\text { Salmonella } \\
\text { Carrau }\end{array}$ & Private home & $53(1)$ & $\begin{array}{l}\text { Cantaloupe, honeydew, } \\
\text { watermelon }\end{array}$ & $\begin{array}{l}\text { PHAC 2009; } \\
\text { Nielsen et al. } \\
2010\end{array}$ \\
\hline 2013 & May & US (MN) & Salmonella & Private home & $7(0)$ & Watermelon & CDC 2018 \\
\hline 2013 & May & US (MN) & Norovirus & $\begin{array}{l}\text { Restaurant-sit- } \\
\text { down dining }\end{array}$ & $59(0)$ & Cantaloupe & CDC 2018 \\
\hline 2017 & January & US (MN) & Norovirus & $\begin{array}{l}\text { Long-term care/ } \\
\text { nursing home/ } \\
\text { assisted-living facility }\end{array}$ & $9(0)$ & Mixed cut fruit & CDC 2018 \\
\hline
\end{tabular}

a Pathogens abbreviated and associated with outbreaks include E. (Escherichia)

${ }^{b}$ NR: Not Reported

*Table was last updated on 5-3-2021. The most recent data published by the CDC indicates that there have been no outbreaks since 2017. 
Table 6. Foodborne illness outbreaks associated with multiple foods (including melons), 2000-2016*

\begin{tabular}{|c|c|c|c|c|c|c|c|}
\hline Year & Month & Location & Pathogen $^{a}$ & Location of Consumption & $\begin{array}{c}\text { Cases } \\
\text { (deaths) }\end{array}$ & Food Vehicle & Reference \\
\hline 2000 & June & US (MN) & Norovirus & $\begin{array}{l}\text { Caterer (food prepared off- } \\
\text { site from where served) }\end{array}$ & $33(0)$ & Sandwich, turkey; cantaloupe & CDC 2018 \\
\hline 2002 & April & US (DC) & $\begin{array}{l}\text { Staphylococcus } \\
\text { aureus }\end{array}$ & Other & $8(0)$ & $\begin{array}{l}\text { Other cheese, pasteurized; } \\
\text { honeydew melon; potato, fried }\end{array}$ & CDC 2018 \\
\hline 2002 & August & US (SC) & $\begin{array}{l}\text { Salmonella } \\
\text { Heidelberg }\end{array}$ & Prison/jail & $26(0)$ & $\begin{array}{l}\text { Watermelon; ground beef, } \\
\text { hamburger; biscuit, unspecified }\end{array}$ & CDC 2018 \\
\hline 2004 & April & US (CO) & Norovirus & $\begin{array}{l}\text { Long-term care/nursing } \\
\text { home/assisted-living } \\
\text { facility }\end{array}$ & $62(0)$ & $\begin{array}{l}\text { Strawberries; melon, unspecified; } \\
\text { house salad }\end{array}$ & CDC 2018 \\
\hline 2005 & March & US (WA) & Norovirus & $\begin{array}{l}\text { Private home/residence; } \\
\text { caterer (food prepared } \\
\text { off-site from where served); } \\
\text { other }\end{array}$ & $20(0)$ & $\begin{array}{l}\text { Dips, unspecified; strawberries; } \\
\text { melon, unspecified }\end{array}$ & CDC 2018 \\
\hline 2005 & May & US (UT) & $\begin{array}{l}\text { Salmonella } \\
\text { Enteritidis }\end{array}$ & Private home/residence & $126(0)$ & $\begin{array}{l}\text { Cantaloupe; chicken, bbq; corned } \\
\text { beef, unspecified }\end{array}$ & CDC 2018 \\
\hline 2005 & July & US (CO) & $\begin{array}{l}\text { Salmonella } \\
\text { Newport }\end{array}$ & Unknown & $24(1)$ & $\begin{array}{l}\text { Cantaloupe; ground beef, } \\
\text { unspecified }\end{array}$ & CDC 2018 \\
\hline 2006 & May & US (CO) & $\begin{array}{l}\text { Salmonella } \\
\text { Typhimurium } \\
\text { var Cope }\end{array}$ & $\begin{array}{l}\text { Restaurant-other or } \\
\text { unknown type }\end{array}$ & $7(0)$ & Chicken; watermelon & CDC 2018 \\
\hline 2006 & August & US (NY) & $\begin{array}{l}\text { Salmonella } \\
\text { Newport }\end{array}$ & Other & $12(0)$ & $\begin{array}{l}\text { Honeydew melon; plum, } \\
\text { unspecified; pizza, unspecified; } \\
\text { cheese, unspecified }\end{array}$ & CDC 2018 \\
\hline 2007 & January & US (CO) & Norovirus & $\begin{array}{l}\text { Banquet facility (food } \\
\text { prepared and served on- } \\
\text { site); grocery store }\end{array}$ & $19(0)$ & Honeydew, caramel rolls & CDC 2018 \\
\hline 2007 & May & US (NJ) & $\begin{array}{l}\text { Salmonella } \\
\text { Litchfield }\end{array}$ & $\begin{array}{l}\text { Restaurant-other or } \\
\text { unknown type }\end{array}$ & $30(0)$ & $\begin{array}{l}\text { Fruit salad; green salad; } \\
\text { cantaloupe; honeydew melon; } \\
\text { grapes, unspecified }\end{array}$ & CDC 2018 \\
\hline 2007 & November & US (IA) & Norovirus & $\begin{array}{l}\text { Restaurant-other or } \\
\text { unknown type; other }\end{array}$ & $44(0)$ & Mixed fruit & CDC 2018 \\
\hline 2008 & July & US (GA) & Norovirus & $\begin{array}{l}\text { Private home/residence; } \\
\text { Caterer (food prepared off- } \\
\text { site from where served) }\end{array}$ & $27(0)$ & $\begin{array}{l}\text { Other cheese, pasteurized; } \\
\text { watermelon; grapes, unspecified }\end{array}$ & CDC 2018 \\
\hline 2008 & August & US (CO) & E. coli O157:H7 & Grocery store; other & $5(0)$ & $\begin{array}{l}\text { Cantaloupe; ground beef, } \\
\text { hamburger }\end{array}$ & CDC 2018 \\
\hline 2009 & February & $\begin{array}{l}\text { US } \\
\text { (multistate) }\end{array}$ & $\begin{array}{l}\text { Salmonella } \\
\text { Carrau }\end{array}$ & Grocery store & $53(1)$ & Melon & CDC 2018 \\
\hline 2009 & July & US (OR) & Norovirus & $\begin{array}{l}\text { Caterer (food prepared off- } \\
\text { site from where served) }\end{array}$ & $18(0)$ & Rolls; watermelon; cheese/roll & CDC 2018 \\
\hline 2010 & February & US (MO) & Norovirus & $\begin{array}{l}\text { Banquet facility (food } \\
\text { prepared and served on- } \\
\text { site) }\end{array}$ & $139(0)$ & Fruit salad & CDC 2018 \\
\hline 2010 & April & US (WY) & Norovirus & $\begin{array}{l}\text { Banquet facility (food } \\
\text { prepared and served on- } \\
\text { site); other }\end{array}$ & $33(0)$ & $\begin{array}{l}\text { Multiple salads; unspecified fish; } \\
\text { couscous; zucchini; mousse; } \\
\text { water; ice; soup, other vegetable- } \\
\text { based; soup, specialty, other; } \\
\text { enchiladas; red salsa; chips, } \\
\text { tortilla; ranch dressing; refried } \\
\text { beans, unspecified; melon, } \\
\text { unspecified; cake; salad dressings; } \\
\text { pork, sweet and sour; mixed } \\
\text { vegetables; egg rolls; banana, } \\
\text { unspecified; cookies }\end{array}$ & CDC 2018 \\
\hline
\end{tabular}




\begin{tabular}{|l|l|l|l|l|l|l|l|}
\hline Year & Month & Location & Pathogen & Location of Consumption & $\begin{array}{c}\text { Cases } \\
\text { (deaths) }\end{array}$ & Food Vehicle \\
\hline 2011 & July & US (MN) & E. coli O157:H7 & Private home/residence & 6 (0) & Fruit & Reference \\
\hline 2014 & June & US (WI) & Norovirus & $\begin{array}{l}\text { Caterer (food prepared off- } \\
\text { site from where served) }\end{array}$ & $21(0)$ & Fruit platter & CDC 2018 \\
\hline 2016 & May & US (NY) & Norovirus & $\begin{array}{l}\text { Banquet facility (food } \\
\text { prepared and served on- } \\
\text { site) }\end{array}$ & 136 (0) & Mixed cut fruit; crudites & CDC 2018 \\
\hline 2016 & July & US (WV) & $\begin{array}{l}\text { Salmonella } \\
\text { Heidelberg }\end{array}$ & Private home/residence & 4 (0) & Chicken; cantaloupe; eggs \\
\hline
\end{tabular}

a Pathogens abbreviated and associated with outbreaks include E. (Escherichia)

*Table was last updated on 5-3-2021. The most recent data published by the CDC indicates that there have been no outbreaks since 2016. 